\title{
Fully depleted back-illuminated p-channel CCD development
}

\author{
C.J. Bebek, J.H. Bercovitz, D.E. Groom, S.E. Holland, R.W. Kadel, A. Karcher, \\ W.F. Kolbe, H.M. Oluseyi, N.P. Palaio, V. Prasad, B.T. Turko, and G. Wang \\ Lawrence Berkeley National Laboratory, 1 Cyclotron Road, Berkeley, CA
}

\begin{abstract}
An overview of CCD development efforts at Lawrence Berkeley National Laboratory is presented. Operation of fully-depleted, back-illuminated CCD's fabricated on high resistivity silicon is described, along with results on the use of such CCD's at ground-based observatories. Radiation damage and point-spread function measurements are described, as well as discussion of CCD fabrication technologies.
\end{abstract}

Keywords: CCD, fully depleted, back illuminated, radiation damage, point spread function, fabrication

\section{INTRODUCTION}

We have developed fully-depleted, back-illuminated CCD imagers for scientific applications. ${ }^{1}$ The CCD's are fabricated on high-resistivity, n-type silicon substrates. The substrate resistivity is greater than about $5000 \Omega$ $\mathrm{cm}$. In addition, a substrate bias is used to independently deplete the substrate, resulting in 200-300 $\mu \mathrm{m}$ thick, fully-depleted CCD's.

Although originally developed for ground-based astronomy, CCD's of this type have several advantages for space applications. The use of a thick substrate improves the near-infrared response and greatly reduces "fringing" in astronomical images that occurs when the absorption length of the incident light exceeds the thickness of the CCD's. Also, the substrate bias used to deplete the CCD allows one to reduce the amount of lateral diffusion of charge carriers that occurs during the transit of photogenerated carriers from their generation point to the CCD collection wells. This effect in conjunction with the thickness chosen for the CCD allows for smaller pixels that have a point-spread function (PSF) that is consistent with the pixel size. The use of smaller pixels allows for a reduced telescope focal length resulting in a more compact telescope for a given imaging area.

The CCD described in this work is p-channel. P-channel CCD's have been shown to be more resistant to the damaging effects of space radiation, including both displacement damage from protons ${ }^{2}$ and ionizing damage to the gate insulators. ${ }^{3}$

Thick CCD's have several drawbacks, however. Cosmic rays will affect more pixels in a thick CCD, a particular concern for space applications. This is obviated somewhat by the use of multiple exposures of the same field, typically dithered anyway to remove CCD artifacts. In addition, depth of focus problems will be an issue for near-infrared light and small $f$-number camera systems. In that case the light rays are incident at large angles to the normal with deep absorption in the silicon, although the high refractive index of silicon helps in this regard by "straightening out" the light.

In this work we present results on the use of this type of CCD at ground-based observatories and describe the development of $4096 \times 2048(15 \mu \mathrm{m}$ pixel $)$ devices for ground-based work. CCD's have been irradiated with high-energy protons and characterized both in terms of charge-transfer efficiency (CTE) and dark current degradation. Identification of the microscopic traps responsible for the degradation has been done with pocket pumping experiments. Results on proton damage and ${ }^{60} \mathrm{Co}$ effects on CCD test transistors will be presented. In addition, measurement of the CCD point-spread function is described, as well as results on CCD fabrication both at Lawrence Berkeley National Laboratory (LBNL) and at a commercial CCD foundry.

Further author information: (Send correspondence to S.E.H.) S.E.H.: E-mail: seholland@lbl.gov, Telephone: 15104865069 


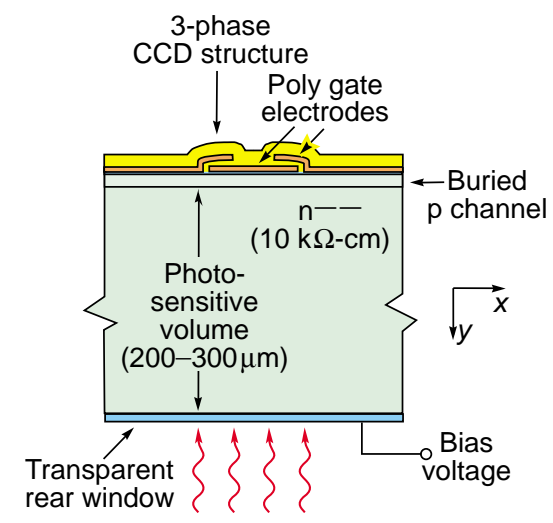

Figure 1. Cross-sectional diagram of the CCD described in this work.

\section{FULLY-DEPLETED CCD PHYSICS AND OPERATION}

Figure 1 shows a cross-sectional diagram of the fully-depleted, back-illuminated CCD. A conventionally-processed, three-phase CCD is fabricated on a high-resistivity, n-type silicon substrate. We have fabricated CCD's on both $100 \mathrm{~mm}$ and $150 \mathrm{~mm}$ diameter high-resistivity silicon substrates. The resistivity of $100 \mathrm{~mm}$ wafers is as high as 10,000-12,000 $\Omega$-cm, while the initial work on $150 \mathrm{~mm}$ wafers has been on 4,000-8,000 $\Omega$-cm silicon.

The thickness of the CCD results in improved near-infrared sensitivity when compared to conventional thinned CCD's. ${ }^{1}$ This is due to the strong dependence of absorption length on wavelength at photon energies approaching the silicon bandgap. ${ }^{4}$ Figure 2 shows measured quantum efficiency (QE) versus wavelength for a fully-depleted, back-illuminated CCD operated at $-130^{\circ} \mathrm{C}$. The QE is especially high at near-infrared wavelengths. The CCD shown in Figure 2 has a two-layer anti-reflection (AR) coating tuned for good red response. It consists of $60 \mathrm{~nm}$ of indium tin oxide (ITO) and $100 \mathrm{~nm}$ of silicon dioxide $\left(\mathrm{SiO}_{2}\right)$.

Thick, fully-depleted CCD's also greatly reduce the problem of "fringing" at near-infrared wavelengths. ${ }^{5}$ Fringing occurs when the absorption depth of the incident light exceeds the CCD thickness. Multiple reflections result in fringing patterns that are especially a problem in 10-20 $\mu$ m thick CCD's used in spectrographs.

A unique feature of the CCD shown in Figure 1 is the use of a substrate bias to fully deplete the substrate. For a thick CCD fabricated on high-resistivity silicon the channel potential is to first order independent of the substrate bias. ${ }^{1}$ This is because for typical substrate thicknesses and doping densities considered here only a small fraction of the electric field lines from the depleted channel terminate in the fully-depleted substrate. Hence the vertical clock levels can be set to optimize operating features such as well capacity and CTE while the substrate bias is used to deplete the substrate.

The substrate bias also plays a role in the point-spread function of the CCD. For light absorbed near the back surface of the CCD the lateral charge spreading during transit of the photogenerated charges through the fully-depleted substrate to the CCD collection wells is described by an rms standard deviation given by ${ }^{1,6}$

$$
\sigma_{o d} \approx \sqrt{2 \frac{k T}{q} \frac{y_{D}^{2}}{\left(V_{\mathrm{sub}}-V_{J}\right)}}
$$

where $k$ is Boltzmann's constant, $T$ is absolute temperature, $q$ is the electron charge, $y_{D}$ is the thickness of the depleted substrate, $V_{\text {sub }}$ is the applied substrate bias voltage, and $V_{J}$ is an average potential near the CCD potential wells due to the channel potentials. $V_{\text {sub }}-V_{J}$ is the voltage drop across the region where the photogenerated holes are drifted by the electric field. This result is a simplified asymptotic form that is independent of the substrate doping and is valid for high electric fields in the substrate. Therefore in this case the PSF is directly proportional to $y_{D}, \sqrt{T}$, and $1 / \sqrt{\left(V_{\text {sub }}-V_{J}\right)}$. The PSF for a CCD of this type can be improved by reducing the substrate thickness and operating the CCD at high substrate bias. PSF measurements are described in more detail in Section 5. 


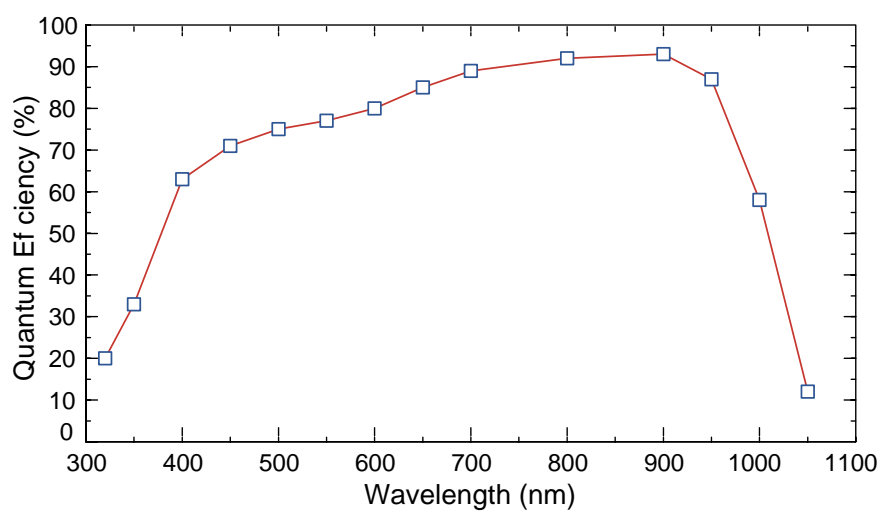

Figure 2. Quantum efficiency measured on a $800 \times 1980,15 \mu \mathrm{m}$ pixel back-illuminated, fully-depleted CCD. The measurement was performed at Lick Observatory and the operating temperature was $-130^{\circ} \mathrm{C}$. The thickness was $\approx 280 \mu \mathrm{m}$.

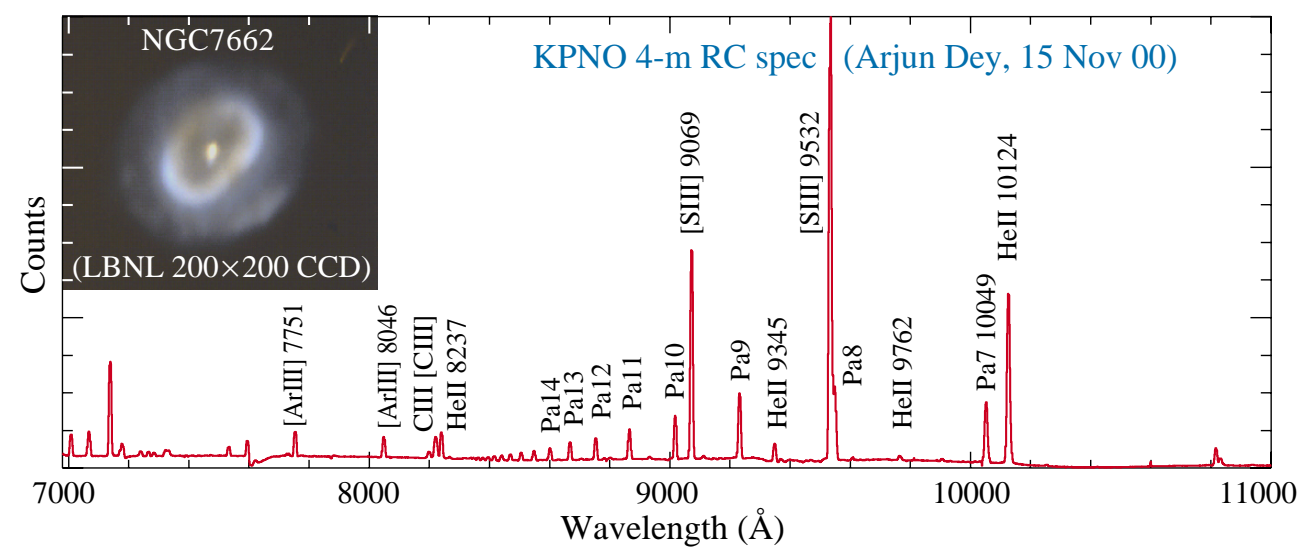

Figure 3. Near-infrared spectrum of NGC7662 measured at the NOAO RC Spectrometer using a $\approx 300-\mu \mathrm{m}$ thick, $800 \times 1980(15 \mu \mathrm{m}$ pixel $)$, fully-depleted, back-illuminated CCD. The image of NGC7662 shown was taken on an early prototype $200 \times 200(15 \mu \mathrm{m}$ pixel $)$ fully-depleted CCD at the Lick Observatory Nickel 1-m telescope.

In the actual implementation of the CCD an insulating AR coating is used and hence the backside bias voltage is actually applied at the front side of the CCD. Floating $\mathrm{p}^{+}$guard rings are used to gradually drop the potential from the substrate bias voltage applied to a front-side substrate contact to a grounded guard ring that surrounds the CCD. ${ }^{1,7}$

\section{RESULTS AT GROUND-BASED OBSERVATORIES}

Back-illuminated CCD's of the type shown in Figure 1 are in use at ground-based observatories. $800 \times 1980$ (15 $\mu \mathrm{m}$ pixel) CCD's are used in spectrographs at the National Optical Astronomy Observatory (NOAO) Kitt Peak National Observatory (KPNO) for enhanced red response while a $2048 \times 2048(15 \mu \mathrm{m}$ pixel $)$ CCD is in use at the Lick Observatory Hamilton Spectrograph. The latter CCD is used primarily for extra-solar planet searches and has an improved PSF when compared to previous back-illuminated CCD's used for this purpose. ${ }^{8}$ This is discussed in more detail in Section 5.

Figure 3 shows a near-infrared spectrum of the planetary nebula NGC7662 taken at the NOAO RC spectrograph using an $800 \times 1980(15 \mu \mathrm{m}$ pixel), fully-depleted, back-illuminated CCD. The image of NGC7662 inset in Figure 3 was taken on an early prototype $200 \times 200(15 \mu \mathrm{m}$ pixel $)$ CCD $^{9}$ at the Lick Observatory Nickel 1-m telescope. As seen in Figure 3 spectral lines out to wavelengths somewhat beyond $1 \mu \mathrm{m}$ are clearly resolved. Obtaining such spectra with conventional, thinned CCD's is very difficult due to fringing and low QE at near-infrared wavelengths. 

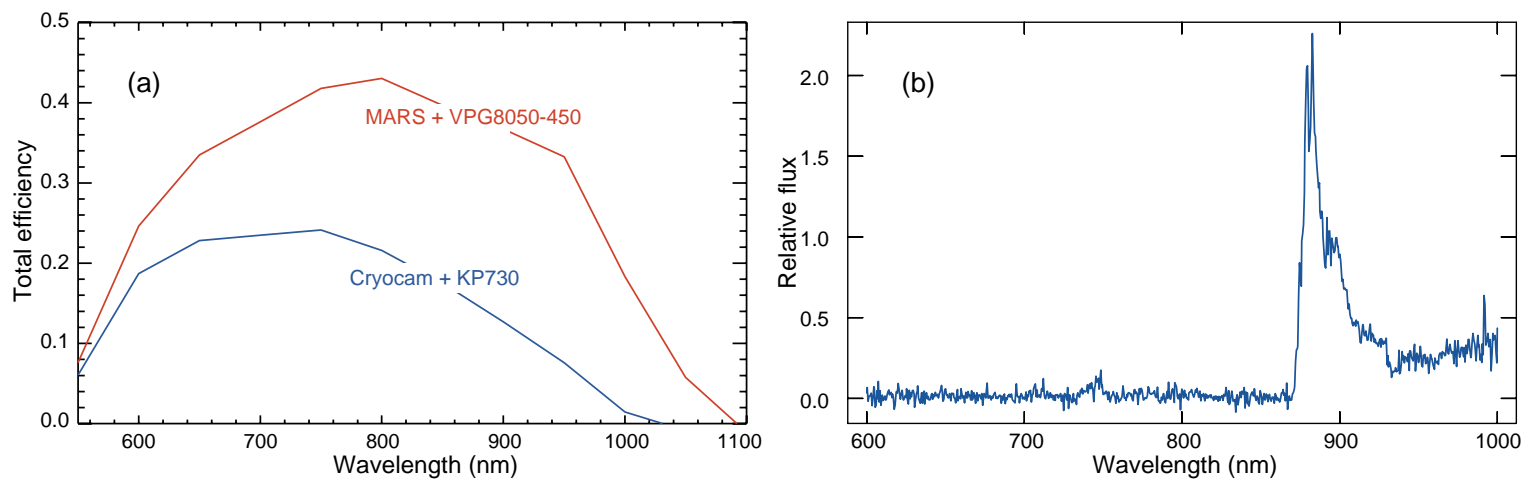

Figure 4. (a) Measured efficiencies of the NOAO Multi-Aperture Spectrometer (MARS) and the Cryocam. MARS is an upgrade of Cryocam. The MARS instrument uses a $800 \times 1980,15 \mu \mathrm{m}$ pixel, fully-depleted, back-illuminated CCD fabricated at LBNL. The improved efficiency at near-IR wavelengths is primarily due to the higher quantum efficiency of the LBNL CCD when compared to the thinned CCD used in the Cryocam. (b) Spectrum of a high-redshift quasar taken with MARS in June 2003. Data courtesy of Xiaohui Fan of the University of Arizona Astronomy Department and the Sloan Digital Sky Survey. ${ }^{11}$

A $800 \times 1980(15 \mu \mathrm{m}$ pixel $)$, fully-depleted, back-illuminated CCD is also in use at the NOAO MultiAperture Red Spectrometer (MARS). ${ }^{10}$ This is a red-optimized, low-to-medium resolution spectrograph that takes advantage of the high near-infrared QE of the back-illuminated, thick CCD. The peak QE as measured at NOAO is $92 \%$ in the $800-900 \mathrm{~nm}$ wavelength regime and fringing is virtually nonexistent. MARS is an upgrade of Cryocam. The total efficiency of both spectrometers is shown in Figure 4(a). In addition to the LBNL CCD, there were decreases in pupil obscuration, improved coatings, and a new grism. However, the improvements in the red were almost entirely due to the LBNL CCD. Figure 4(b) shows a science result from MARS, a spectrum of a high-redshift quasar. For wavelengths exceeding $900 \mathrm{~nm}$ the spectrum taken at the KPNO 4-m telescope with MARS is as good as that taken at a 6-8 m class telescope with the same exposure time. ${ }^{12}$

We are developing $4096 \times 2048,15 \mu \mathrm{m}$ pixel CCD's for use in ground-based astronomy. Characterization tests have been carried out in the LBNL CCD Production Facility utilizing our 4-side abutable mosaic packaging. A total of four devices have been tested to date. The shorts yield on these devices was $100 \%$, while the cosmetics yield was $25 \%$. Table 1 gives the measured values for the cosmetics, read noise, dark current, serial and parallel CTE's, linearity and well depth at $30 \mathrm{kpixel} / \mathrm{sec}$ readout rate for the device with acceptable cosmetics. Note that the read noise is somewhat higher than previously reported ${ }^{1}$ due to the measured device having an early non-optimized transistor geometry.

The standard method of measuring the CTE of front illuminated and thinned back illuminated CCD's is done using ${ }^{55} \mathrm{Fe} \mathrm{x}$-ray sources. However, this method is inadequate for measuring the CTE of $300 \mu \mathrm{m}$ thick back illuminated devices since the $5.9 \mathrm{keV} \mathrm{Mn} \mathrm{K \alpha}$ photon emitted from this source is absorbed $\approx 30 \mu \mathrm{m}$ into the silicon. Lateral diffusion of the photogenerated carriers during drift from the generation point to the CCD potential wells yields very few or no single-pixel events, a necessary condition for the successful application of this method. Therefore, we have characterized our devices using a ${ }^{109} \mathrm{Cd}(22 \mathrm{keV})$ x-ray source and also by using the extended pixel edge response (EPER) method. ${ }^{13}$ The data of Table 1 were generated with the EPER method.

\section{PROTON AND ${ }^{60}$ CO IRRADIATION RESULTS}

Because CCD's destined for space applications are expected to encounter significant doses of radiation exposure during their lifetime, it is important to characterize their radiation tolerance. The radiation is dominated at near-earth orbits by the Van Allen radiation belts and at further distances by the solar proton flux. Both ionizing and non-ionizing energy losses are important contributors to the damage.

Ionizing radiation produces e-h pairs in the CCD that leads ${ }^{13}$ to charge deposition in the dielectric layers insulating the gate electrodes used in clocking and charge readout. This trapped charge causes flat-band effects 
Table 1. Measured performance of a $4096 \times 2048,15 \mu \mathrm{m}$ pixel CCD fabricated at LBNL and operated at $-140^{\circ} \mathrm{C}$.

\begin{tabular}{|c|c|}
\hline Parameter & Performance \\
\hline Cosmetics & 0 hot columns \\
\hline Read noise & $4.5 \mathrm{e}^{-}$ \\
\hline Dark current & $7 \mathrm{e}^{-} / \mathrm{pixel} / \mathrm{hr}$ \\
\hline Parallel CTE & 0.999995 \\
\hline Serial CTE & 0.999995 \\
\hline Linearity & Better than $1 \%$ \\
\hline Well depth & $160,000 \mathrm{e}^{-}$ \\
\hline
\end{tabular}

that alter the operating potentials of the device. We have examined this effect on test structures incorporating MOS capacitors and p-channel FETs similar to those used in the actual CCD's. In these measurements the test structures were exposed at room temperature to increasing doses of ${ }^{60} \mathrm{Co} \gamma$-rays at a radiation facility at LBNL. The room-temperature measurements on transistors similar to those used in the CCD output amplifier show flat-band voltage shifts of $\approx 90-100$ and $\approx 280-300 \mathrm{mV}$ after 5 and $20 \mathrm{krad}$ of ${ }^{60}$ Co $\gamma$-rays. No degradation in subthreshold slope was observed, indicating no significant generation of interface states at these dose levels. It was pointed out by Saks et al. ${ }^{3}$ that in p-channel, buried-channel CCD's with $\mathrm{Si}_{3} \mathrm{~N}_{4}-\mathrm{SiO}_{2}$ gate dielectrics, under normal bias conditions, trapped holes in the oxide from e-h pair production are compensated by electrons trapped in the $\mathrm{Si}_{3} \mathrm{~N}_{4}$ resulting in a lower flat-band voltage shift than in the case of an n-channel, buried-channel CCD. These preliminary measurements were performed with zero bias voltage applied to the device electrodes. Additional tests are currently underway employing CCD's that will be irradiated under normal operating bias conditions.

Proton irradiation is known $^{13}$ to produce displacement damage in silicon through non-ionizing energy loss (NIEL) processes that result in the formation of trapping sites in the material. In p-channel CCD's, hole traps in the channel region with energies close to the valence band can capture charge during readout and degrade the CTE. In addition, midband traps in the depletion region can lead to an increase in dark current.

In conventional CCD's, the phosphorus doped n-channel is susceptible to the generation during irradiation of phosphorus-vacancy (PV) traps ${ }^{14}$ that degrade the CTE whereas, in p-channel devices, the dominant trap is believed to be the divacancy $(\mathrm{VV}){ }^{2}, 15 \mathrm{VV}$ trap formation in irradiated p-channel CCD's is considered to be less likely than PV trap formation in n-channel CCD's. In addition the VV trap energy (0.20 eV above the valence band) lies further from mid-band than PV traps ( $0.45 \mathrm{eV}$ below the conduction band) and should therefore contribute less to the dark current. ${ }^{2,14}$ These properties combine to yield a p-channel CCD that is more radiation resistant than typical n-channel devices.

A number of CCD's were irradiated at room temperature using the LBNL 88-inch cyclotron at $12 \mathrm{MeV}$ to yield a high damage rate at reasonable doses and to achieve a relatively uniform damage level throughout the thickness of the device. The CCD's tested are $512 \times 1024$ pixels, $15 \mu \mathrm{m}$ pitch engineering grade devices and were not backside processed. They are $600 \mu \mathrm{m}$ thick and cannot be fully depleted so were operated as front-side illuminated devices. Irradiation doses ranging from $5 \times 10^{9}$ to $1 \times 10^{11}$ protons $/ \mathrm{cm}^{2}$ were used.

Measurements of the CTE and dark current were performed as a function of temperature on the irradiated CCD's. Figure 5(a) shows the CTE degradation as a function of dose at a measurement temperature of 128 K, chosen to be near optimum for these devices. The error bars shown represent the uncertainty in the widths of the x-ray lines in the corresponding stacking plots used in the calculation. Figure 5(b) shows the CTE as a function of temperature for the highest dose measured in these experiments. The difference between the serial and parallel CTE is striking and is a result of the different clocking speeds used in the two cases. As discussed in our earlier paper ${ }^{16}$ the observed CTE degradation as a function of dose converted to $\mathrm{MeV} / \mathrm{g}$ is more than an order of magnitude smaller than that found for typical n-channel CCD's. 


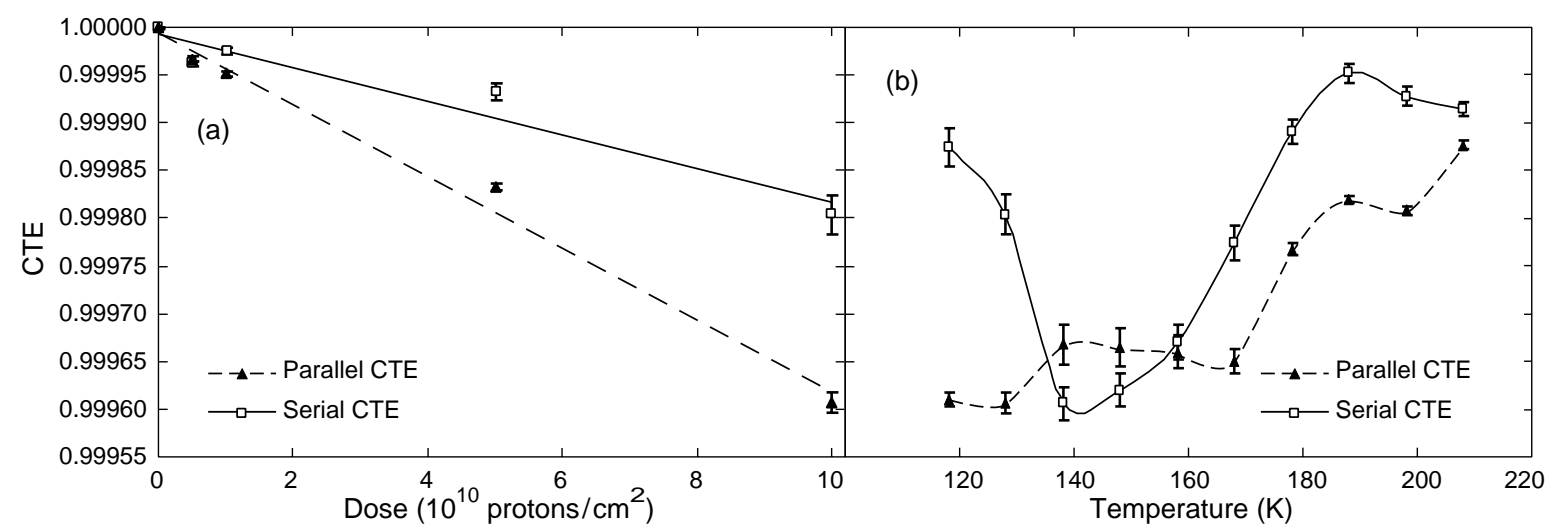

Figure 5. (a) CTE degradation as a function of proton dose measured at 128K. (b) Temperature dependence of CTE at a dose of $1 \times 10^{11}$ protons $/ \mathrm{cm}^{2}$.
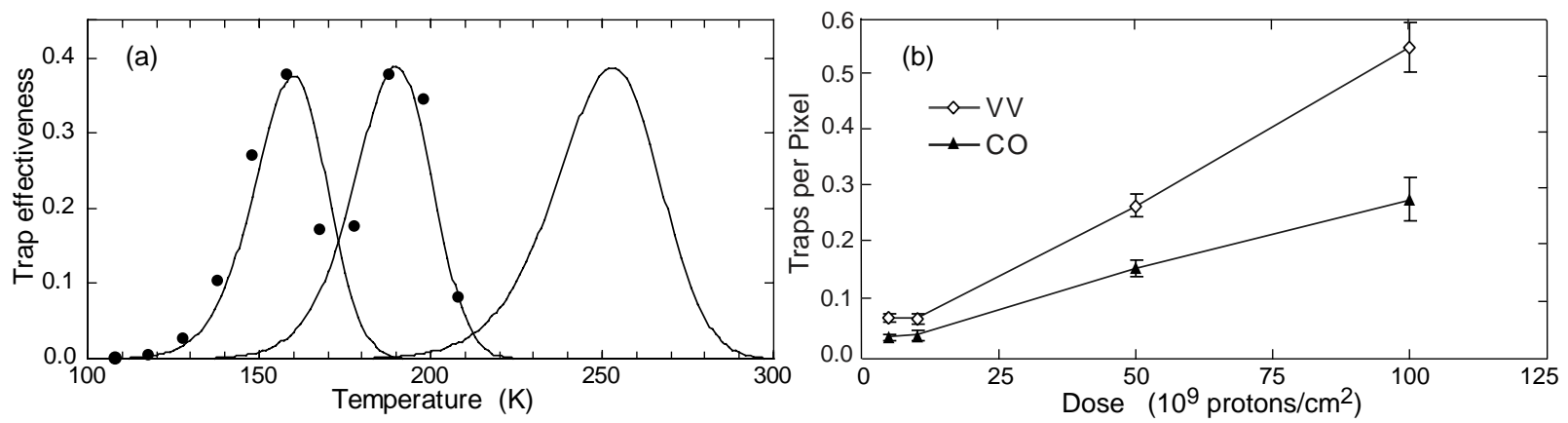

Figure 6. (a) Measured trap depth (closed circles) together with computed trap effectiveness for 3 kinds of hole traps: VV with a peak at $160 \mathrm{~K}, \mathrm{Ci}$ at $190 \mathrm{~K}$ and $\mathrm{CO}$ at $253 \mathrm{~K}$. The dose was $1 \times 10^{10}$ protons $/ \mathrm{cm}^{2}$. (b) Proton dose dependence of the fitted trap densities $\mathrm{VV}$ and $\mathrm{CO}$.

It is clear from Figure 5 that the observed CTE behavior is strongly affected by the nature of the radiation induced traps, the operating temperature, and the details of the clocking used to read out the CCD. In order to identify and characterize the traps present we used pocket pumping. ${ }^{17}$ In this method ${ }^{13}$ the CCD is first illuminated with a uniform source of light to produce a flat field exposure. Then, before readout, the accumulated charge is moved back and forth multiple times. In this process, traps that are effective at the temperature and clocking rate used cause the uniform field to be drawn into characteristic peaks and valleys that can be used to locate the traps and identify their characteristics.

Figure 6(a) shows the trap effectiveness (closed circles) as a function of temperature measured one day after irradiation at a dose of $1 \times 10^{10}$ protons $/ \mathrm{cm}^{2}$. Also shown in the figure are computed trap effectiveness for 3 different candidate hole traps, ${ }^{18}$ the divacancy (VV) with peak at $160 \mathrm{~K}$, carbon interstitial $(\mathrm{Ci})$ at $190 \mathrm{~K}$ and the carbon-oxygen $(\mathrm{CO})$ at $253 \mathrm{~K}$. The close agreement with experiment enables us to identify the two measured peaks as the first two candidates above. The higher temperature peak at $190 \mathrm{~K}$ became very weak after a few days, indicating that this kind of trap was annealing rapidly. The CO peak was not detected in the pocket pumping experiments due to large dark currents at higher temperatures, but was found necessary to obtain realistic fits to the observed temperature dependent CTE measurements. All of these traps have been studied extensively ${ }^{19}$ in investigations of n-type high resistivity silicon used in high-energy physics experiments. 
A number of authors ${ }^{2,13}$ have used the Shockley-Hall-Reed ${ }^{20}$ theory to develop models for the degradation of the CTE as a function of trap density. In these models the CTE is a function of the temperature-dependent trapping and detrapping times and the serial or parallel clock overlap times used during the charge readout. Using a modified model ${ }^{17}$ appropriate to our clocking scheme, we fit the measured CTE data as a function of dose and extracted the trap densities shown in Figure 6(b). While all three of the predicted traps were included in the fit, the VV and CO traps were found to be the dominant contributors to the CTE degradation. By modeling the effects of radiation damage in this fashion it is possible to predict the expected CTE under various irradiation doses expected in the space environment and perhaps to tune the fabrication and wafer materials selection to make even more radiation resistant CCD's in the future.

\section{MEASUREMENT AND CHARACTERIZATION OF POINT-SPREAD FUNCTION}

The point spread function (PSF) measurement is based on the Foucault knife-edge technique. However, instead of placing a physical knife edge in the beam, we select a grid of pixels on the CCD (integration region) and measure the variation of the total charge in the grid as the beam is scanned across the grid. Since the beam is contained within the grid of pixels at the start of the scan and lies outside the grid of pixels at the end of it, the variation of the total charge in the grid as a function of the beam position yields the integral of the beam profile in the scan direction. The advantage of this technique over other PSF measurement techniques is that the point spread function is independent of the pixel size and there is no scattering from a physical knife edge.

To characterize the point spread function, we require a light-tight, thermally stable, evacuated environment for the CCD. The CCD is placed in a standard IR-labs dewar that has been fitted with a thin, wide-band AR coated window and a mechanical shutter. The dewar is evacuated to $\sim 10^{-7}$ torr, and the CCD is cooled to $-140^{\circ} \mathrm{C}$ using a cold finger which is in contact with the back of the CCD as well as a bath of liquid nitrogen.

A well-collimated and intense beam is needed to reduce the effect of any beam dispersion that is not caused by the lateral diffusion in the CCD and decrease the statistical uncertainties in the measurement. To achieve this, we use a high-intensity General Electric light bulb and place it behind a multi-lens collimator system. The collimated light beam passes through a filter wheel consisting of a set of Corion $70 \mathrm{~nm}$ bandwidth interference filters between $450 \mathrm{~nm}$ and $650 \mathrm{~nm}$. The beam is focussed using a convex filter onto an optical fiber which transmits the light to a pinhole projector. The pinhole projector is made up of a 10-inch brass tube which encloses a $10 \mu \mathrm{m}$ pinhole and a 5x-long-working-distance Mitutoyo microscope objective. The inner surface of the tube is threaded and darkened to reduce the effect of light re-scattering into the collimated beam. Light-tight rubber bellows are placed in between the projector and the dewar to prevent ambient light from reaching the CCD.

The pinhole projector is mounted onto an $x-y$ - $z$ translation stage, where the $x-y$ plane has been carefully aligned to the CCD plane, with the $x$-axis parallel and $y$-axis perpendicular to the CCD rows. The translation stage and the dewar are placed on a Newport suspension table which provides mechanical stability.

Motion of the projector in the $x$ - and $y$-directions is carried out using a stepper motor with a step size of $0.4 \mu \mathrm{m}$, and the position of the projector is read out using a linear optical encoder with an accuracy of about $0.1 \mu \mathrm{m}$. Movement in the $z$-direction is performed manually and is needed to focus the beam onto the CCD.

The CCD is read out using an ACS GenII controller that has been modified to supply the substrate bias voltage and the data is processed using a SUN workstation.

The beam profile in the row and column-directions were determined by focusing the beam onto the CCD and then moving the pinhole projector in 1.2-1.4 $\mu \mathrm{m}$ steps in the $x$ - and $y$-directions, respectively. After each step, an image of the beam was obtained by opening the shutter for $0.5-3 \mathrm{~s}$. The shutter exposure time and lamp intensity were optimized to minimize statistical uncertainties while ensuring that there were no saturated pixels. The images were processed, correcting for inter-pixel gain variations (typically $5 \%$ ) and pedestal levels and fluctuations $(\sim 0.5 \%)$. To account for lamp-intensity and sub-pixel response variations $(\sim 5 \%)$, the total charge in the integration region was normalized to the total charge collected on the CCD. To determine the beam profile width, $\sigma_{\mathrm{CCD}}$, we fitted the rate of variation of the charge in the integration region with respect to the beam position. We used a $1 / \cosh$ form at low substrate bias voltages (partially depleted region, $\leq 14 \mathrm{~V}$ ) 

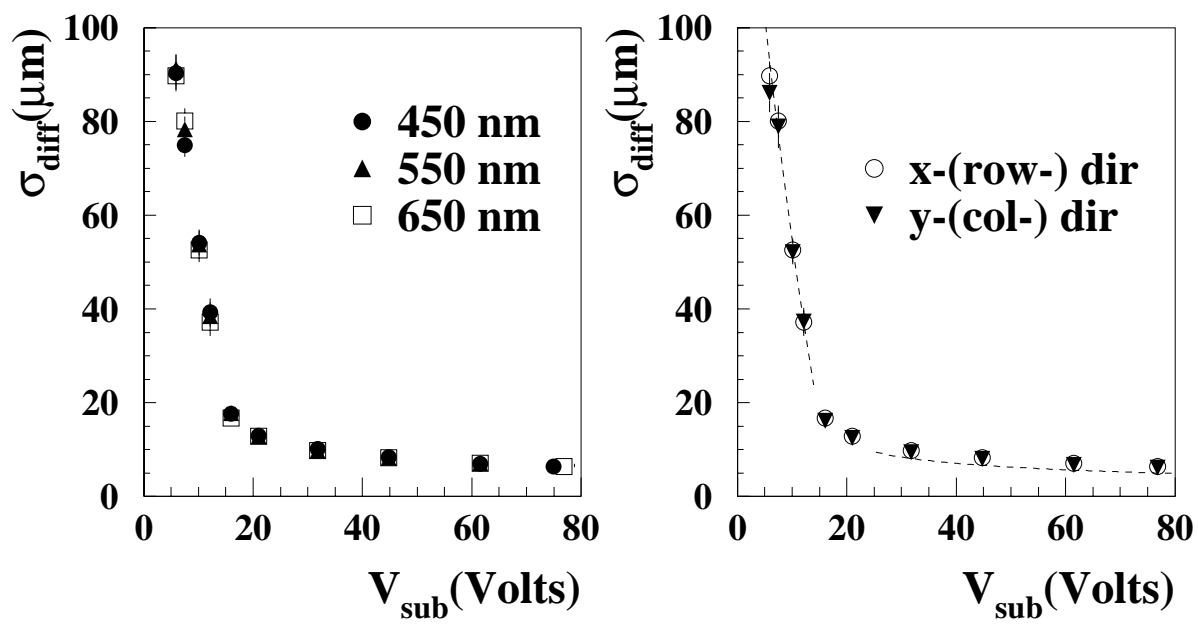

Figure 7. Variation of $\operatorname{PSF}\left(\sigma_{\text {diff }}\right)$ with substrate bias voltage $\left(V_{\text {sub }}\right)$ at $450 \mathrm{~nm}, 550 \mathrm{~nm}$ and $650 \mathrm{~nm}$ in the $x$-(row) direction. Right: Variation of $\operatorname{PSF}\left(\sigma_{\text {diff }}\right)$ with substrate bias voltage $\left(V_{\text {sub }}\right)$ at $650 \mathrm{~nm}$ in the $x$-(row) and $y$-(column) directions. The dashed curves indicate the theoretical fit based on the one-dimensional analytical model. ${ }^{1}$ The error bars in both plots include both statistical and systematic uncertainties.

to account for the wide tails in the beam profile, and a Gaussian at higher substrate voltages (fully-depleted region). ${ }^{1} \sigma_{\mathrm{CCD}}$ is defined such that $68 \%$ of the beam lies within $\pm \sigma_{\mathrm{CCD}}$ of the beam center.

To extract the PSF, $\sigma_{\text {diff }}$, from $\sigma_{\mathrm{CCD}}$, we subtracted the intrinsic beam width, $\sigma_{\text {beam }}$, from $\sigma_{\mathrm{CCD}}$ in quadrature. $\sigma_{\text {beam }}$ was measured separately using the pinhole projector, a knife edge and a photomultiplier and was found to be $1.3 \pm 0.1 \mu \mathrm{m}$. Since $\sigma_{\text {beam }}$ is much smaller than the PSF, the PSF measurement is fairly insensitive to the uncertainties in the intrinsic beam size.

The lateral charge diffusion, $\sigma_{\text {diff }}$, was measured for a $800 \times 1100$ (15 $\mu \mathrm{m}$ pixel), $280 \mu \mathrm{m}$ thick, back-illuminated CCD fabricated at LBNL. The PSF was determined at 10 different substrate bias voltages between $6 \mathrm{~V}$ and $80 \mathrm{~V}$ and at 3 wavelengths: $450 \mathrm{~nm}, 550 \mathrm{~nm}$ and $650 \mathrm{~nm}$. Scans were performed in all four $( \pm x$ - and $\pm y$-) directions over a $200 \times 200$-pixel region of the CCD. Systematic studies included the variation of $\sigma_{\text {diff }}$ with the scan position, direction and integration region size, as well as the determination of the encoder accuracy.

The results of the PSF measurement are shown in Figure 7. We observe that the mean $\sigma_{\text {diff }}$ is $(89.9 \pm 3.0) \mu \mathrm{m}$ and $(6.38 \pm 0.24) \mu \mathrm{m}$ at a substrate bias voltage of $6 \mathrm{~V}$ and $77 \mathrm{~V}$, respectively. $\sigma_{\text {diff }}$ is seen to decrease rapidly with increasing substrate voltage in the partially-depleted region and approaches the constant-field result (Equation 1) at high substrate voltages. We do not observe any significant difference in $\sigma_{\text {diff }}$ at wavelengths between $450 \mathrm{~nm}$ and $650 \mathrm{~nm}$ nor between $x$ - and $y$-scan directions. The former is not surprising since the difference between the absorption lengths in silicon at $450 \mathrm{~nm}$ and $650 \mathrm{~nm}$ is only $2.7 \mu \mathrm{m}$.

Figure 7 also shows the fit to the data based on the one-dimensional analytical model. ${ }^{1}$ Based on the fit, we determine the donor density, $N_{D}$, in the silicon substrate to be $3 \times 10^{11} / \mathrm{cm}^{3}(\approx 14.3 \mathrm{k} \Omega$-cm) and the effective junction potential, $V_{J}$, to be about $-1.7 \mathrm{~V}$.

A $200 \mu \mathrm{m}$ thick LBNL CCD with $15 \mu \mathrm{m}$ pixels is in use at the Lick 3-m Coudé spectrometer. The point spread function (PSF), rather than the red sensitivity, is of interest to the planet-search group, which looks for small periodic variations in radial velocity in nearby sun-like stars. The variations are caused by the star's motion as the star and the planet rotate about a common center of mass. The period of the planet is obtained directly from the period of the variations. If the star's mass is estimated from its spectral type, then the radius of the planet's orbit can be deduced. Information about the planetary mass is then inferred from the amplitude. The search is limited by the accuracy with which the stellar radial velocity can be determined, and an important factor in this uncertainty is the PSF of the CCD. Measurements for a case near the sensitivity limit of the observations are shown in Fig. 8. The 2001 measurements were made with a "conventional" thinned CCD, and the nightly rms 


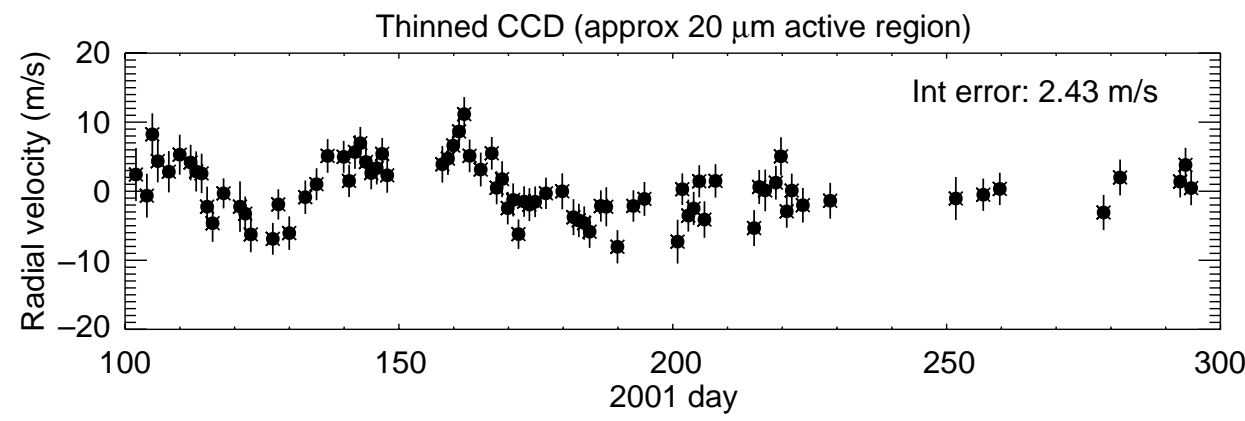

Totally depleted LBNL CCD $(200 \mu \mathrm{m}$ active region)

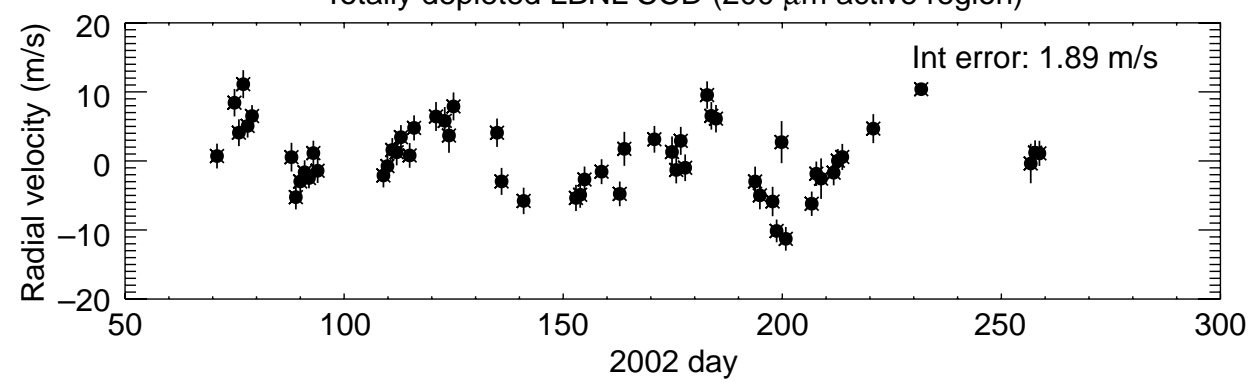

Figure 8. Measured radial velocities in an extra-solar planet study at Lick Observatory comparing a conventional thinned CCD to a $200 \mu \mathrm{m}$ thick LBNL CCD. A Fourier analysis of radial velocities obtained between 1997-1999 for a bright nearby star showed power at 60 days. However, the $2.5 \mathrm{~m} / \mathrm{s}$ velocity precision obtained with a conventional thinned CCD provided an ambiguous result. The narrower PSF of the high-resistivity CCD yielded a $0.6 \mathrm{~m} / \mathrm{s}$ improvement in the velocities in the 2002 season and represents the highest Doppler precision achieved for stars on current planet search surveys. ${ }^{8}$

uncertainty was $2.43 \mathrm{~m} / \mathrm{s}$. Starting with the 2002 season the LBNL CCD, operated over depleted at $40 \mathrm{~V}$, was used. The rms uncertainty was reduced to $1.89 \mathrm{~m} / \mathrm{s}$, very much increasing the confidence limit that a signal with about a 60 day period and a $4 \mathrm{~m} / \mathrm{s}$ amplitude is present. Further improvements can be expected with smaller pixels and a higher bias voltage. These preliminary data were made available to us by Dr. Debra Fischer of the University of California at Berkeley.

\section{CCD FABRICATION}

The CCD's used to date in ground-based observatories were fabricated at the LBNL Microsystems Laboratory, a Class 10 clean room that includes lithography, etching, thin film deposition, and thermal oxidation and annealing equipment for processing $100 \mathrm{~mm}$ diameter wafers. All process steps necessary to fabricate CCD's are done at the Microsystem Laboratory except for ion implantations that are performed at commercial companies.

The LBNL Microsystems Laboratory is primarily a research and development facility capable of small production runs. In order to enhance the production capabilities of LBNL CCD's we have been working with DALSA Semiconductor ${ }^{21}$ to produce fully-depleted, back-illuminated CCD's on $150 \mathrm{~mm}$ substrates.

Working with a commercial CCD foundry has several advantages. The upgrade from $100 \mathrm{~mm}$ to $150 \mathrm{~mm}$ wafers results in more than a factor of two increase in available wafer area. Figure 9 shows a $100 \mathrm{~mm}$ diameter wafer fabricated at LBNL as well as a $150 \mathrm{~mm}$ wafer fabricated at DALSA Semiconductor. Included on both wafers are 4096 x $2048(15 \mu \mathrm{m})$ CCD's as well as smaller pixel CCD's.

Another advantage of CCD fabrication at a modern $150 \mathrm{~mm}$ wafer foundry is that the fabrication yield is typically increased due to the use of newer, more advanced fabrication equipment. Also, rapid fabrication cycles allow for quick verification of new CCD design concepts. In addition, more aggressive design rules result in smaller parasitic capacitances in output amplifier structures and potentially higher performance CCD's.

In order to get a preliminary indication of fabrication yield at DALSA Semiconductor, 10 large-area CCD's were packaged and tested in vacuum dewars at cryogenic temperatures (typically $-140^{\circ} \mathrm{C}$ ). No prescreening 

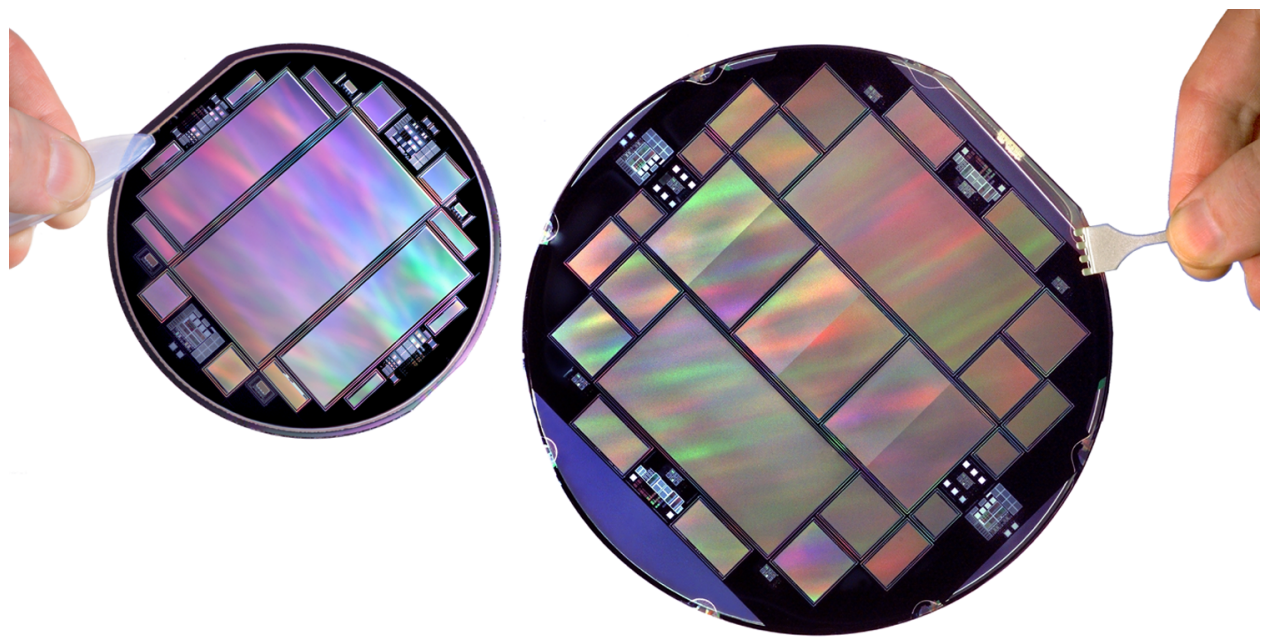

Figure 9. Comparison of a $100 \mathrm{~mm}$ wafer fabricated at LBNL with a $150 \mathrm{~mm}$ wafer fabricated at DALSA Semiconductor. The large-area devices are $4096 \times 2048,15 \mu \mathrm{m}$ CCD's.

of any type was done before the CCD's were packaged. The CCD's are of similar imaging area $(\approx 3 \mathrm{~cm}$ by $3 \mathrm{~cm}$ ) and are either $2880^{2}\left(10.5 \mu \mathrm{m}\right.$ pixel) or $2520^{2}(12 \mu \mathrm{m}$ pixel). The majority of the CCD's were operated front-illuminated and were not fully depleted given the substrate thickness of 600-700 $\mu \mathrm{m}$. Two CCD's were thinned to $300 \mu \mathrm{m}$ and were packaged for back illumination. Table 2 shows the test results of packaged CCD's from various DALSA fabrication lots and wafers. Of the 10 CCD's packaged and tested, 7 were found to be of high quality.

Table 2. Table showing the results of the packaging and testing of 10 large-area CCD's fabricated at DALSA Semiconductor. $\mathrm{N}$ denotes that the CCD had a notch implant in the vertical register, while BI denotes the CCD was processed and packaged for back illumination. CCD's from two separate fabrication lots are included. The 5 digit number in the CCD Name column is the lot number and the following number is the wafer number.

\begin{tabular}{|c|c|c|}
\hline CCD Name & Size & Results \\
\hline $75091-18$ & $2880 \times 2880(10.5 \mu \mathrm{m})$ & Nearly perfect \\
\hline $75091-6$ & $2880 \times 2880(10.5 \mu \mathrm{m})$ & Works well \\
\hline $75091-24$ BI & $2520 \times 2520(12 \mu \mathrm{m})$ & Not working \\
\hline $75091-24$ BI N & $2520 \times 2520(12 \mu \mathrm{m})$ & Good, a few warm columns \\
\hline $75091-17$ & $2880 \times 2880(10.5 \mu \mathrm{m})$ & One half working but with hot columns \\
\hline $75091-5$ & $2880 \times 2880(10.5 \mu \mathrm{m})$ & Nearly perfect \\
\hline $75091-17$ & $2520 \times 2520(12 \mu \mathrm{m})$ & Works well \\
\hline $75091-5$ & $2520 \times 2520(12 \mu \mathrm{m})$ & Works well, 1 amplifier not working \\
\hline $81481-1 \mathrm{~N}$ & $2880 \times 2880(10.5 \mu \mathrm{m})$ & Works well \\
\hline $81481-2 \mathrm{~N}$ & $2880 \times 2880(10.5 \mu \mathrm{m})$ & Vertical clock short on one side \\
\hline
\end{tabular}

Figure 10 shows an image taken with CCD 75091-24 (notch implant, back illuminated) which is the 4th entry of Table 2. The image was generated by illumination through a $35 \mathrm{~mm}$ photographic negative. The 6.35 Mpixel CCD was back illuminated and operated fully depleted with a substrate bias voltage of $80 \mathrm{~V}$. The operating temperature was $-140^{\circ} \mathrm{C}$. This CCD was entirely fabricated at DALSA Semiconductor with the exception of the deposition of AR coatings, which were done at LBNL. Other than for several non-saturated column defects, the CCD has very good cosmetics. 


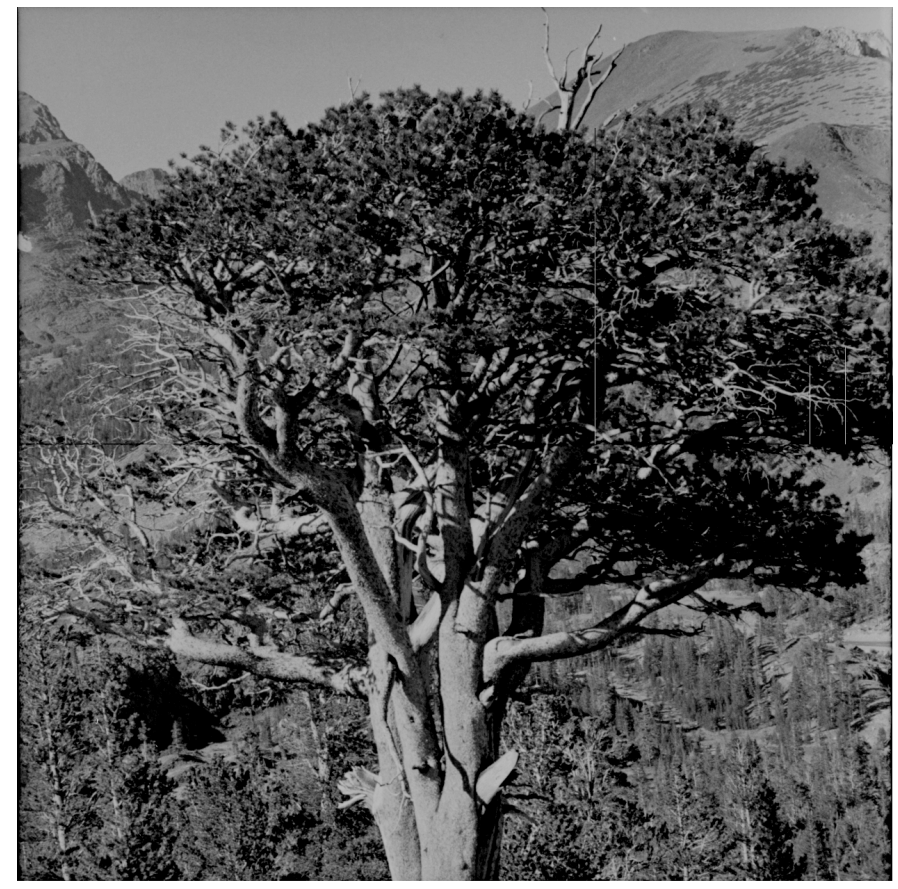

Figure 10. Back-illuminated CCD test pattern image taken on a $2520^{2}$ (12 $\mu \mathrm{m}$ pixel) fully-depleted, back-illuminated CCD. The CCD was operated at $-140^{\circ} \mathrm{C}$ and is $300 \mu \mathrm{m}$ thick.

The back-illuminated CCD that produced the image shown in Figure 10 was $300 \mu \mathrm{m}$ thick. The desire to go to thinner CCD's for space applications has led to the realization that significant process development is required in order to reliably produce fully-depleted, back-illuminated CCD's on $150 \mathrm{~mm}$ wafers. As a result the development effort for the processing of non-standard thickness wafers is is now done primarily at LBNL.

A challenge in the production of fully-depleted CCD's is the realization of a back-illumination technology that can withstand fully-depleted operation. This implies that the dark current should not be significantly affected when the depletion region approaches the back side of the wafer.

The back-illumination technology used in the CCD's described in this paper consists of a thin, backside ohmic contact formed by deposition of in-situ doped polycrystalline (ISDP) silicon. ${ }^{1,22}$ This technique has been shown to be capable of maintaining low dark current for over-depleted operation. However, this is a high-temperature step $\left(\approx 650^{\circ} \mathrm{C}\right)$ that is not compatible with conventional aluminum metalization. Therefore some processing must be done on thinned wafers with our technology.

Our approach is to process standard thickness CCD wafers at DALSA Semiconductor for most of the fabrication steps, taking advantage of the benefits mentioned earlier of working with a commercial $150 \mathrm{~mm}$ wafer foundry. Once the CCD's are thinned the final processing is done at LBNL. Under this "business model", CCD's are fabricated on standard thickness $150 \mathrm{~mm}$ wafers through the first 8 masks in the process at DALSA Semiconductor. The wafers are then thinned to $200-250 \mu \mathrm{m}$ with the remainder of the processing performed at LBNL. This includes deposition of the ISDP film, contact and metal masking and etching, and deposition of backside AR coatings.

Challenges in this mode of operation include maintaining sufficient wafer flatness after thinning for subsequent lithography steps as well as realization of robust wafers that do not break in automated wafer handling equipment. Also, subsurface damage that may result from the thinning process ${ }^{23}$ must be eliminated in order to maintain low dark current in fully-depleted operation.

Figure 11(a) shows an image taken on a fully-depleted, back-illuminated $1636 \times 1560(9 \mu \mathrm{m}$ pixel $)$ CCD that is one of the first CCD's fabricated under the new "business model". The CCD thickness is $250 \mu \mathrm{m}$. Shown in 


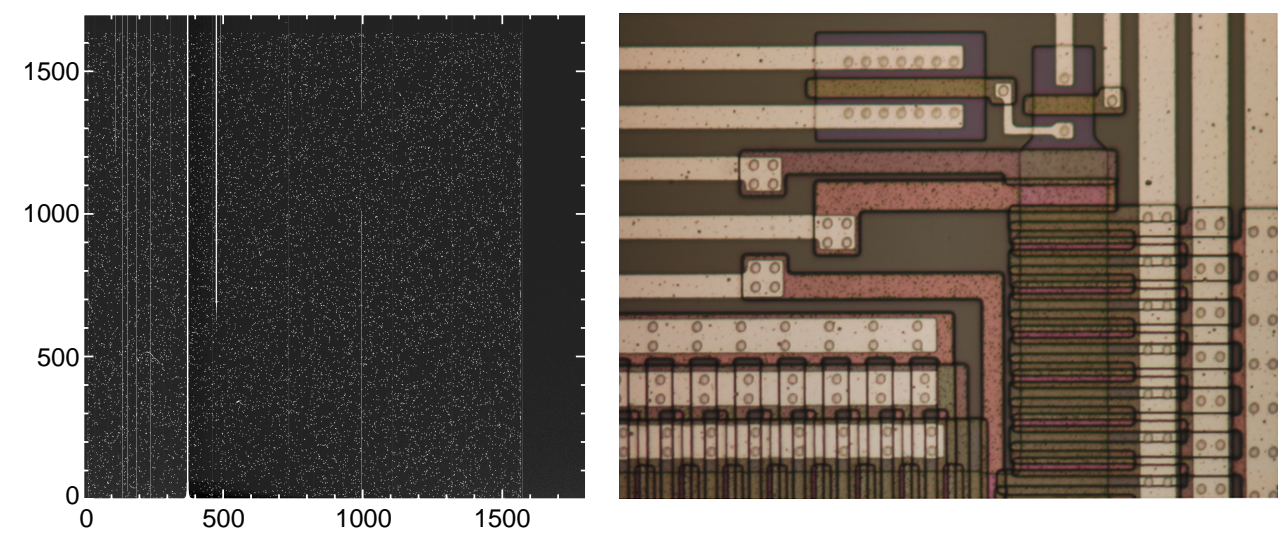

Figure 11. (a) 30 second ${ }^{55} \mathrm{Fe}$ x-ray image taken on a fully-depleted, back-illuminated, $1636 \times 1560$ (9 $\mu \mathrm{m}$ pixel) CCD. The CCD was operated at $-150^{\circ} \mathrm{C}$ and is $250 \mu \mathrm{m}$ thick. (b) Photograph of a portion of a CCD fabricated in the "business model" described in the text.

Figure $11(\mathrm{a})$ is a 30 second exposure of the $\mathrm{CCD}$ to ${ }^{55} \mathrm{Fe} \mathrm{x}$ rays. The $\mathrm{CCD}$ is operated at $50 \mathrm{~V}$ substrate bias, which is well above the depletion voltage for this device. The CCD has a few column defects that were likely introduced during the processing after thinning. This result is encouraging although much development work remains. Figure 11(b) is a photograph of a portion of a CCD fabricated under the "business model" described earlier, demonstrating the good registration of the metal and contact layers (done at LBNL) to the underlying polysilicon and active layers (done at DALSA Semiconductor).

\section{SUMMARY}

We have fabricated p-channel, back-illuminated CCD's that use a substrate bias to achieve full depletion of 200-300 $\mu \mathrm{m}$-thick, high-resistivity silicon substrates. Improved performance over conventional CCD's in terms of near-infrared response and PSF has been demonstrated in instruments at the KPNO 4-m and Lick 3-m telescopes. Proton damage and the effects of ${ }^{60} \mathrm{Co}$ ionizing radiation have been studied, demonstrating superior radiation hardness as compared to n-channel devices. Measurement of the CCD PSF using a virtual knife edge has been described, as well as initial fabrication results of back-illuminated CCD's on $150 \mathrm{~mm}$ wafers in partnership with a commercial foundry.

The CCD technology described in this work is promising for space-based telescopes due to the improved near-infrared response, radiation hardness, and PSF when compared to conventional CCD's. However, in order to take advantage of these benefits, careful system design is required in order to account for the increased pixel loss due to cosmic rays.

\section{ACKNOWLEDGEMENTS}

This work was supported by DOE contract No. DE-AC03-76SF00098. Kitt Peak National Observatory and the National Optical Astronomy Observatory are operated by the Association of Universities for Research in Astronomy, Inc. (AURA) under cooperative agreement with the National Science Foundation. The Sloan Digital Sky Survey is managed by the Astrophysical Research Consortium (ARC) for the Participating Institutions. The Participating Institutions are The University of Chicago, Fermilab, the Institute for Advanced Study, the Japan Participation Group, The John Hopkins University, Los Alamos National Laboratory, the Max-Planck-Institute for Astronomy (MPIA), the Max-Planck-Institute for Astrophysics (MPA), New Mexico State University, University of Pittsburg, Princeton University, the United States Naval Observatory, and the University of Washington.

The authors gratefully acknowledge CCD performance data provided by Arjun Dey, Xiaohui Fan and Debra Fischer, and CCD development work done at Lick Observatory by Richard Stover, Mingzhi Wei, Kirk Gilmore, and Bill Brown. 


\section{REFERENCES}

1. S.E. Holland, D.E. Groom, N.P. Palaio, R.J. Stover, and M. Wei, "Fully depleted, back-illuminated chargecoupled devices fabricated on high-resistivity silicon," IEEE. Trans. Elec. Dev., 50, pp. 225-238, 2003.

2. J.P. Spratt, B.C. Passenheim, R.E. Leadon, "The effects of nuclear radiation on P-channel CCD imagers," in 1997 IEEE Radiation Effects Data Workshop, NSREC Snowmass 1997, Workshop Record. Held in conjunction with IEEE Nuclear and Space Radiation Effects Conference.

3. N.S. Saks, J.M. Killiany, P.R. Reid, and W.D. Baker, "A radiation hard MNOS CCD for low temperature applications," IEEE Trans. Nucl. Sci., 26, pp. 5074-5079, 1979.

4. W.C. Dash and R. Newman, "Intrinsic optical absorption in single-crystal germanium and silicon at $77 \mathrm{~K}$ and 300K," Phys. Rev., 99, pp. 1151-1155, 1955.

5. D.E. Groom et al., "Quantum efficiency of a back-illuminated CCD imager: an optical approach," in Proc. SPIE, 3649, pp. 80-90, 1999.

6. S.E. Holland et al., "Development of back-illuminated, fully-depleted CCD image sensors for use in astronomy and astrophysics," presented at the 1997 IEEE Workshop on Charge-Coupled Devices and Advanced Image Sensors, Bruges, Belgium, 1997.

7. U.S. Patent 6,259,085 "Fully depleted back illuminated CCD," July 10th, 2001.

8. Personal communication, Debra Fischer, University of California-Berkeley Astronomy Department.

9. S.E. Holland et al., "A $200 \times 200 \mathrm{CCD}$ image sensor fabricated on high-resistivity silicon," in IEDM Technical Digest, pp. 911-914, 1996.

10. http://www.noao.edu/kpno/mars/

11. Sloan Digital Sky Survey, http://www.sdss.org.

12. Personal communication, Xiaohui Fan, University of Arizona Astronomy Department and Sloan Digital Sky Survey.

13. J.R. Janesick, Scientific charge-coupled devices, SPIE Press, Bellingham, WA, 2001.

14. J. Janesick, G. Soli, T. Elliot, and S. Collins, "The effects of proton damage on charge-coupled devices," in Proc. SPIE, 1447, pp. 87-108, 1991.

15. G.R. Hopkinson, "Proton damage effects on p-channel CCDs," IEEE Trans. Nucl. Sci., 46, pp. 1790-1796, 1999.

16. C.J. Bebek et al., "Proton radiation damage in p-channel CCDs fabricated on high-resistivity silicon," IEEE Trans. Nucl. Sci., 49, pp. 1221-1225, 2002.

17. C.J. Bebek et al., "Proton radiation damage in high-resistivity n-type silicon CCD's," in Proc. SPIE, 4669, pp. 161-171, 2002.

18. M. Moll, "Radiation damage in silicon particle detectors," Ph.D. dissertation, Physics Dept., Univ. of Hamburg, Germany, 1999.

19. M. Bruzzi, "Radiation damage in silicon detectors for high-energy physics experiments," IEEE Trans. Nucl. Sci., 48, pp. 960-971, 2001.

20. W. Shockley and W.T. Read, Jr. "Statistics of the recombinations of holes and electrons," Phys. Rev., 87, pp. 835-842, 1952.

21. http://www.dalsasemi.com, DALSA Semiconductor, Bromont, Quebec, Canada.

22. S.E. Holland, N.W. Wang, and W.W. Moses, "Development of low noise, back-side illuminated silicon photodiode arrays," IEEE Trans. Nucl. Sci., 44, pp. 443-447, 1997.

23. L.-Q. Chen, X. Zhang, T.-Y. Zhang, H.Y. Lin, and S. Lee, "Micro-Raman spectral analysis of the subsurface damage layer in machined silicon wafers," J. Mater. Res., 15, pp. 1441-1444, 2000. 